\title{
Mesenteritis esclerosante: presentación de caso clínico, síntomas, causas y tratamiento
}

\author{
Bernal Delgado \\ chavel09@hotmail.com \\ ORCID: https://orcid.org/0000-0003-0141-323X \\ Universidad Laica Eloy Alfaro de Manabí \\ Dra. María José Castro Garcia \\ mjcgar0@gmail.com \\ ORCID: https://orcid.org/0000-0002-6058-1084 \\ Dr. Jean Paul Santana Vera \\ jeanpa0594@gmail.com \\ ORCID: https://orcid.org/0000-0002-2984-8377 \\ Universidad Laica Eloy Alfaro de Manabí \\ Dra. Katherine Monserrate Arcentales Mero \\ kathymonserrate@hotmail.com \\ ORCID: https://orcid.org/0000-0003-3394-0982 \\ Universidad Laica Eloy Alfaro de Manabí
}

\section{RESUMEN}

La mesenteritis esclerosante es una enfermedad no neoplásica poco común que afecta el mesenterio del intestino delgado con inflamación fibrosante crónica, hay pocos datos sobre la historia natural y las opciones terapéuticas para esta afección La mesenteritis esclerosante forma parte de un espectro de condiciones idiopáticas, de rara presentación, caracterizada por inflamación crónica del mesenterio y fibrosis, y fue descrita por primera vez en 1924.

El método empleado para desarrollar el estudio, es la presentación de un caso clínico.

El objetivo propuesto de este escrito, es conocer las diferentes formas de presentación de un cuadro clínico de mesenteritis.

Entre los resultados importantes encontrados se describen los siguientes: La localización más frecuente es el mesenterio del intestino delgado, pero en algunos casos puede afectar al mesocolon (sigmoides). La evolución histológica progresa en 3 estadios. El primero es la lipodistrofia mesentérica en la que los macrófagos invaden el tejido mesentérico. En este estadio el cuadro es oligosintomático y de progreso benigno. El segundo es la PM en la que se observa la presencia de infiltrado de células inflamatorias plasmáticas y algunos polimorfonucleares, células gigantes de cuerpo 
extraño y macrófagos.

Las principales conclusiones sobre el estudio, la SM es un trastorno idiopático poco común que afecta el mesenterio del intestino delgado con diversos grados de fibrosis, inflamación y necrosis grasa. Su presentación clínica es bastante variable y varía de asintomática a enfermedad debilitante. Aproximadamente el $50 \%$ de los pacientes podría no necesitar ningún tratamiento.

Palabras claves. paniculitis mesentérica; ilion; enfermedad de weber- Christian; emesis 


\title{
Sclerosing mesenteritis: clinical case presentation, symptoms, causes and treatment
}

\begin{abstract}
Sclerosing mesenteritis is a rare non-neoplastic disease that affects the mesentery of the small intestine with chronic fibrosing inflammation, there is little data on the natural history and therapeutic options for this condition Sclerosing mesenteritis is part of a spectrum of idiopathic conditions, rare presentation, characterized by chronic inflammation of the mesentery and fibrosis, and was first described in 1924.

The method used to develop the study is the presentation of a clinical case.

The proposed objective of this writing is to know the different forms of presentation of a clinical picture of mesenteritis.

Among the important results found, the following are described: The most frequent location is the mesentery of the small intestine, but in some cases it can affect the mesocolon (sigmoid). The histological evolution progresses in 3 stages. The first is mesenteric lipodystrophy in which macrophages invade the mesenteric tissue. At this stage the picture is oligosymptomatic and of benign progress. The second is the PM in which the presence of infiltrate of plasma inflammatory cells and some polymorphonuclear cells, foreign body giant cells and macrophages is observed.

The main conclusions about the study, MS is a rare idiopathic disorder that affects the mesentery of the small intestine with varying degrees of fibrosis, inflammation and fat necrosis. Its clinical presentation is quite variable, ranging from asymptomatic to debilitating disease. About $50 \%$ of patients may not need any treatment.
\end{abstract}

Keywords. mesenteric panniculitis; ilium; weber-christian disease; emesis

Artículo recibido: 10 Agosto. 2021 Aceptado para publicación: 07. Setiembre. 2021 Correspondencia: chavel_09@hotmail.com Conflictos de Interés: Ninguna que declarar 


\section{INTRODUCCIÓN}

El término mesenteritis esclerosante, hace referencia a una enfermedad idiopática, rara, de curso crónico, caracterizada por cambios inflamatorios y fibróticos en el tejido adiposo del mesenterio del intestino. Según Sharma en su revisión sistemática, fue descrita por primera vez por Jura en 1924 como mesenteritis retráctil; posteriormente Crane, en 1955, presenta cinco casos con mesenterio intestinal firme y engrosado, y Ogden en 1960 la reseña como una paniculitis mesentérica. Con el advenimiento de la tomografía, Mindelzun describe los hallazgos tomográficos conocidos como "mesenterio brumoso" y en 1980, Cherayil analiza la composición lipídica del mesenterio ( Sharma, Yadav, Marie Needham, \& Feuerstadt, 2017)

La mesenteritis esclerosante (ME) es una enfermedad rara que afecta al mesenterio de etiología desconocida, pero se cree que puede ser el resultado de una respuesta inflamatoria inespecífica del mesenterio a una agresión alérgica, química, térmica, infecciosa, autoinmune o quirúrgica, aunque no existe evidencia científica que sostenga esta hipótesis. Es un proceso benigno, que en la mayoría de los casos se autolimita. Pocos pacientes requieren tratamiento quirúrgico, el cual generalmente está dirigido a complicaciones secundarias como obstrucción intestinal.

El término de ME engloba diferentes denominaciones de! mismo proceso idiopático inflamatorio de los mesos, que se utilizan en la literatura médica para referirse a las distintas variables histológicas de la misma entidad entre los que se incluyen: lipodistrofia mesentérica, mesenteritis retráctil, enfermedad de Weber-Christian, mesenteritis xantulogranulomatosa y paniculitis nodular sistémica ( Labalde Martíneza, y otros, 2016). Aunque la clínica y los hallazgos radiológicos pueden hacer sospechar la enfermedad, su diagnóstico definitivo exige una confirmación histológica mediante biopsia.

No se conocen estudios que determinen la prevalencia de la enfermedad en la población general, pero en algunos se estima la frecuencia de la mesenteritis esclerosante en grandes series de pacientes consecutivos, a los que se les realizó tomografía por otros motivos. Se ha relacionado con una gran variedad de causas como cirugía abdominal previa, trauma, causas autoinmunitarias o patología oncológica, que puede estar presente al momento del diagnóstico o desarrollarse después del mismo; el linfoma, es la neoplasia más frecuentemente asociada. 


\section{Síntomas}

La mesenteritis esclerosante ocurre generalmente entre la $6^{\mathrm{a}}$ y $7^{\mathrm{a}}$ década de la vida. Es más frecuente en hombres, esta enfermedad es asintomática en un $43 \%$ y el diagnóstico frecuentemente se realiza mediante la exploración abdominal, cuando se presenta síntomas, estos pueden corresponder a dolor abdominal, anorexia, náuseas, hipertermia, diarrea y baja de peso, en ocasiones se puede presentar con masa palpable única o múltiple y en forma excepcional se manifiesta como sangrado rectorragia, ictericia, obstrucción gástrica, e incluso abdomen agudo.

La obstrucción intestinal, tanto de intestino delgado como de colon, raramente se ha reportado como una complicación de la enfermedad y ocurre con mayor frecuencia en presencia de mesenteritis retráctil. La duración de los síntomas varía en el tiempo desde su inicio desde las 2 semanas hasta 16 años, con un promedio de 6 meses. Alrededor del $72 \%$ de los pacientes han presentado síntomas por menos de un año, los hallazgos al examen físico no han sido bien definidos, siendo más frecuente la presencia de una o varias masas profundas en el cuadrante abdominal superior izquierdo.

\section{Diagnóstico}

La mesenteritis esclerosante tiene un pronóstico favorable y puede resolverse en forma espontánea. En casos asintomáticos puede no requerir tratamiento y mantenerse en observación. En pacientes con síntomas leves, algunos autores recomiendan observar, mientras otros sugieren terapia inmunosupresora agresiva para prevenir progresión de la lesión. Se han utilizado esteroides, logrando disminuir la inflamación y mejorar el curso de la enfermedad, principalmente en aquellos casos en que los hallazgos histológicos muestran una respuesta inflamatoria con mínima fibrosis. En casos de falta de respuesta con prednisona, se dispone de otras alternativas como ciclofosfamida, azatioprina, colchicina con respuestas favorables en algunos casos. Se han utilizado también la progesterona y el tamoxifeno, indicadas probablemente por sus propiedades fibrinolíticas, pero la progesterona se ha asociado a recurrencia de los síntomas.

\section{Los exámenes y procedimientos utilizados para diagnosticar la mesenteritis esclerosante incluyen los siguientes:}

- Exploración física. Durante una exploración física, el médico buscará pistas que puedan ayudar a determinar tu diagnóstico. Por ejemplo, la mesenteritis esclerosante 
a menudo forma una masa en la parte superior del abdomen que se puede sentir durante una exploración física.

- Pruebas de diagnóstico por imágenes. Las pruebas de diagnóstico por imágenes del abdomen pueden revelar mesenteritis esclerosante. Las pruebas de diagnóstico por imágenes pueden incluir tomografía computarizada (TAC) o resonancia magnética (MRI, por sus siglas en inglés).

- Extraer una muestra de tejido para su análisis (biopsia). Si estás experimentando signos y síntomas de mesenteritis esclerosante, el médico puede recomendar una biopsia para descartar otras enfermedades y hacer un diagnóstico definitivo. Se puede tomar una muestra de biopsia insertando una aguja larga a través de la piel o durante la cirugía. Antes de comenzar el tratamiento, los médicos harán una biopsia para confirmar el diagnóstico y descartar otras posibilidades, incluidos ciertos tipos de cáncer como el linfoma y el carcinoide (Navarro \& Rivas Rivas, 2018)

\section{Tratamiento}

Podrían diagnosticarse la mesenteritis esclerosante mientras se recibe atención para otra afección. Si no se experimenta sintomatología. En su lugar, el médico puede recomendar pruebas de diagnóstico por imágenes periódicas para controlar tu afección.

El tratamiento médico para la mesenteritis esclerosante tienen por objetivo controlar la inflamación. Los medicamentos pueden comprender los siguientes:

- Corticoesteroides. Los corticoesteroides como la prednisona controlan la inflamación. Los corticoesteroides se pueden usar solos, pero generalmente se combinan con otros medicamentos. Generalmente no se toman más de tres o cuatro meses debido a los efectos secundarios.

- Terapia hormonal. Los tratamientos hormonales como el tamoxifeno pueden retardar el crecimiento del tejido cicatricial. El tamoxifeno (Soltamox) se suele combinar con corticoesteroides $\mathrm{u}$ otros medicamentos y se puede utilizar a largo plazo. El tamoxifeno aumenta el riesgo de coágulos sanguíneos y normalmente se combina con una aspirina diaria para reducir este riesgo. La progesterona (Prometrium) se puede utilizar como una alternativa al tamoxifeno, pero también tiene efectos secundarios significativos. 
- Otros medicamentos. Se han utilizado varios otros medicamentos para tratar la mesenteritis esclerosante, como azatioprina (Imuran, Azasan), colchicina (Colcrys, Mitigare), ciclofosfamida y talidomida (Talomida).

\section{Cirugía}

Si la mesenteritis esclerosante avanza hasta bloquear el flujo de alimentos a través del sistema digestivo, es posible que necesites cirugía para eliminar la obstrucción.

\section{DESARROLLO}

\section{Presentación del caso}

Se trata de un paciente de sexo masculino de 74 años de edad, con antecedentes patológicos personales de asma bronquial, enfermedad coronaria preexistente, insuficiencia suprarrenal aguda por consumo prolongado de corticoides para el tratamiento del asma. No presentaba hábitos tóxicos. Consultó con su médico de cabecera por presentar dolor abdominal de intensidad moderada, periumbilical, no irradiado, de inicio brusco y repentino, que coincidió con la ingesta de diclofenaco por una mialgia. Al examen físico céfalo caudal, el abdomen estaba levemente distendido, sin defensa ni reacción peritoneal, con ruidos hidroaéreos positivos. No presentó náuseas, vómitos, diarrea ni hipertermia.

Fue tratado como una gastritis aguda medicamentosa con $40 \mathrm{mg}$ diarios de omeprazol. Consultó nuevamente con su médico, por continuar con el mismo cuadro abdominal. Se decidió su ingreso hospitalario para vigilancia y tratamiento médico, se le realizaron estudios cardíacos dado sus antecedentes (enzimas cardíacas y electrocardiograma normales). Al cuarto día de hospitalización se evidencia un incremento del dolor abdominal, siempre periumbilical, con poca respuesta a analgésicos comunes. Se le realizó exámenes de laboratorio en el que se objetivó leucocitosis (36.300 por mm3) con neutrofilia y eritrosedimentación de $86 \mathrm{~mm} / \mathrm{h}$. Se realizó una ecografía abdominopelvica, dejando en evidencia una imagen inflamatoria en el mesenterio sin distensión de asas abdominales. Se transfirió al paciente a una unidad hospitalaria de mayor complejidad. Al quinto día, se recibe en el servicio de cirugía, continuando con dolor abdominal periumbilical de gran intensidad y escasa respuesta a los analgésicos comunes, sin irradiación, con leve distensión abdominal, ruidos hidroaéreos positivos, sin defensa ni reacción peritoneal, con catarsis positiva sin diarrea, con buena tolerancia oral y afebril. Al ingreso presentó un laboratorio con 28.700 leucocitos por mm3, $95 \%$ 
de neutrófilos, eritrosedimentación de $98 \mathrm{~mm} / \mathrm{h}$, proteína C reactiva de $31,8 \mathrm{mg} \%$ (valor normal hasta 0,05 mg\%), y LDH de 676 U/l. Se realizó una tomografía computada (TC) con contraste oral y endovenoso (Figuras 1 y 2) que informó aumento de la densidad del tejido graso en la región mesentérica paramediana izquierda, con densidad de partes blandas, con aspecto inflamatorio difuso, englobando estructuras arteriales y venosas, pero sin compresión de las mismas y sin alteración de las asas de intestino delgado adyacentes. Estos hallazgos fueron compatibles con PM.

Figura 1. Tomografía computada, corte sagital (día 5)

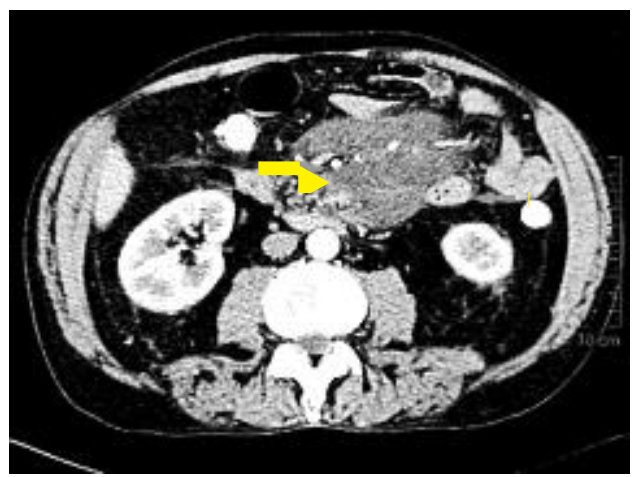

Aumento de la densidad del tejido graso mesentérico, difuso, que respeta la arteria mesentérica y las asas de intestino delgado (flechas).
Figura 2. Tomografía computada, corte axial (día 5)

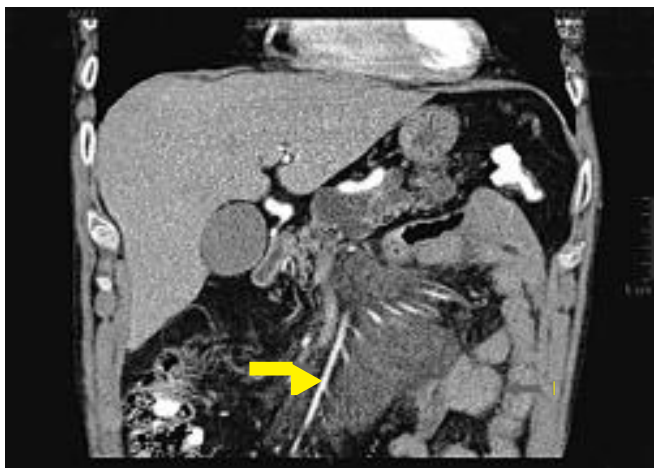

Se observa la indemnidad de la arteria mesentérica en todo su trayecto y de las asas intestinales (flechas).

Con este diagnóstico se decidió su internación con hidratación parenteral, dieta oral, morfina por vía endovenosa y antibióticos de amplio espectro a mayor eficacia. Al sexto día el paciente continuó afebril, con leve mejoría del cuadro abdominal. Presentó disminución de la leucocitosis (21.400 por $\mathrm{mm} 3$ con $65 \%$ de neutrófilos) y proteína C reactiva de 28,3 $\mathrm{mg} \%$. El paciente continuó internado hasta el décimo día, fecha en que fue dado de alta asintomático, con 14.000 leucocitos por $\mathrm{mm} 3$ y una proteína $\mathrm{C}$ reactiva de $16,3 \mathrm{mg} \%$, indicándosele analgesia por vía oral a demanda y control por consulta externa. El día 18 se realizó una Tomografía Axial Computarizada de control (Figuras 3 y 4) que informó persistencia de la lesión ocupante de región mesentérica con leve retracción y aspecto más sólido y polilobulado. En conjunto con el servicio de diagnóstico por imágenes se decidió realizar el control de la evolución y una punción biopsia en el próximo estudio para confirmación de diagnóstico. El día 28 se realizó una nueva TC (Figura 5) que informó ausencia de imágenes nodulares, con leve retracción 
de asas de intestino delgado, por lo cual no se realizó la punción prevista. El paciente continuó asintomático y se le dio de alta con controles de los 2 primeros semestres.

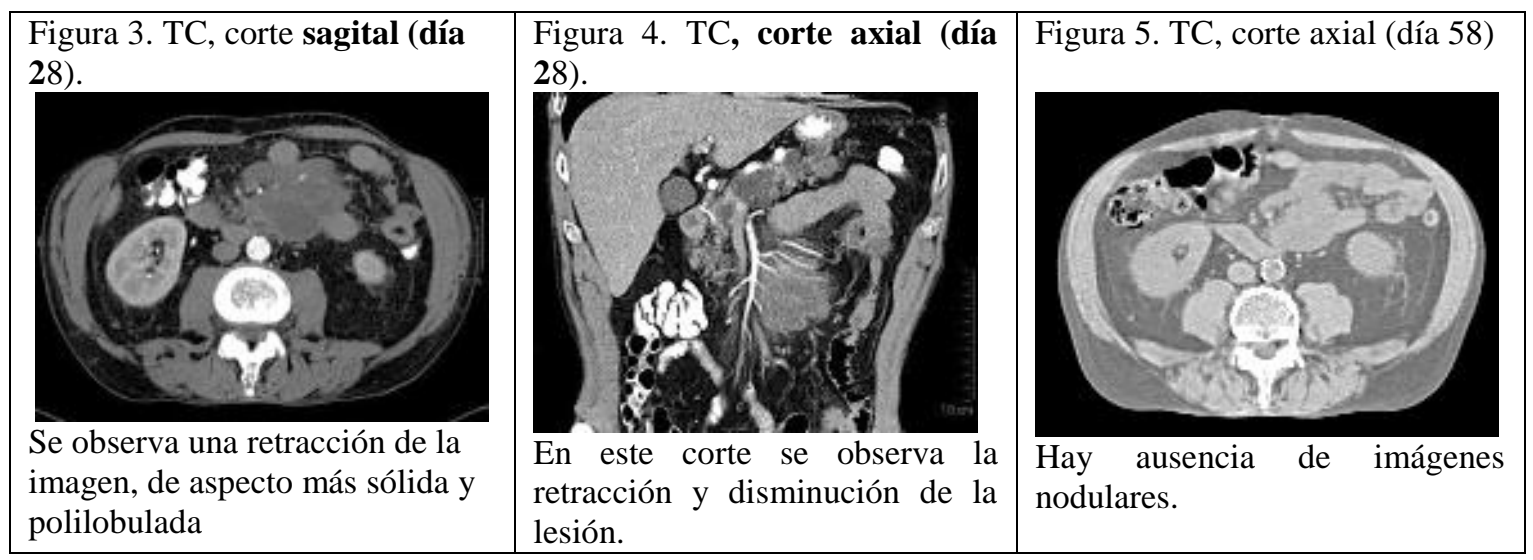

\section{DISCUSIÓN}

La PM es una rara enfermedad que afecta predominantemente a la población masculina (1,8 a 1). Hasta el año 2011 se encontraron 213 casos reportados en la literatura, predominantemente en Estados Unidos, Japón y Francia. ( Poniachik \& Smok S, 2000) La incidencia aumenta con la edad, habiendo pocos casos publicados en niños, probablemente debido a que los mismos presentan menos tejido adiposo en el mesenterio. (Delgado Plasencia , Rodríguez Ballester, López Tomassetti , A Hernández Morales, \& Carrillo Pallarés , 2007) La etiología es incierta, siendo diversas las causas probables (trauma, infecciones, cirugía, etc). Emory y col reportaron una serie en la que en 4 de 84 pacientes había historia de trauma o cirugía. ( Emory, Monihan, \& Carr , 2017) La forma retráctil ha sido descripta en relación a patologías malignas como linfoma, cáncer de pulmón, melanoma, cáncer de colon y tumor carcinoide y se muestra relación con el tabaco. ( Aguilar Chalacán, Vela Benalcazar, Recalde, \& Negrete, 2021) La localización más frecuente es el mesenterio del intestino delgado, pero en algunos casos puede afectar al mesocolon (sigmoides). La evolución histológica progresa en 3 estadios. (Sánchez, Albertini, Cárdenas, Pérez, \& Bécker, 2002) El primero es la lipodistrofia mesentérica en la que los macrófagos invaden el tejido mesentérico. En este estadio el cuadro es oligosintomático y de progreso benigno. El segundo es la PM en la que se observa la presencia de infiltrado de células inflamatorias plasmáticas y algunos polimorfonucleares, células gigantes de cuerpo extraño y macrófagos. Aquí se puede objetivar hipertermia, dolor y distención abdominal y decaimiento. En el tercer 
estadio -la mesenteritis esclerosante- predominan los depósitos de colágeno, la fibrosis y la inflamación. Clínicamente se objetiva una masa palpable y cuadros oclusivos por retracción intestinal. En la mayoría de los pacientes coexisten la necrosis grasa, la inflamación crónica y la fibrosis. Macroscópicamente se puede presentar de tres formas según la clasificación, tipo 1 o forma difusa, tipo 2 o forma aislada, y tipo 3 o múltiple. Esta última es la de presentación menos frecuente. El síntoma más frecuente es el dolor abdominal de moderada intensidad en la mayoría de las veces y con relativa respuesta a los analgésicos, como fue en nuestro caso.

Otros síntomas de presentación son náuseas, alteración del ritmo intestinal, pérdida de peso, enterorragia e hipertermia. La sintomatología tiene una duración variable, de semanas a años y su progreso puede ser continuo o intermitente e inclusive estar ausente. Al examen físico el hallazgo más frecuente es la palpación de una tumoración abdominal en la región periumbilical. Los estudios de laboratorio son a veces normales y con cambios inespecíficos, pudiendo encontrarse como único hallazgo aumento de la eritrosedimentación y la proteína $\mathrm{C}$ reactiva, y anemia en los casos más evolucionado. Los estudios imagenológicos son de gran utilidad, la radiografía simple de abdomen puede presentar cambios inespecíficos, así como los estudios contrastados, la ecografía puede objetivar cambios hiperecogénicos a nivel de la grasa intestinal, pero estos cambios son también inespecíficos, la TC de abdomen es de gran utilidad, siendo ésta a veces suficiente para el diagnóstico, el principal cambio que se observa en la TC es el aumento de la densidad del tejido graso mesentérico en forma de masa, que rodea a los vasos sanguíneos sin afectarlos, esta masa generalmente se ubica del lado izquierdo, afectando al yeyuno, el signo del "halo graso", un halo de menor densidad que rodea a los ganglios y vasos dentro de la misma masa, fue reportado en el $75 \%$ a $85 \%$ de los pacientes con PM, aunque también puede verse en pacientes con linfoma no-Hodgkin. En aproximadamente el 55\% de los pacientes con PM se objetiva una "seudocápsula tumoral", engrosamiento seudocapsular que rodea a la masa y la separa del tejido adyacente. La presencia de nódulos, usualmente de menos de $5 \mathrm{~mm}$ de diámetro, fue encontrada en aproximadamente el $80 \%$ de los pacientes con PM y generalmente corresponden a ganglios linfáticos. Cuando se objetivan nódulos mayores de $10 \mathrm{~mm}$ son atípicos de PM y deben, por lo tanto, ser sometidos a una biopsia percutánea para descartar patología maligna ( Akram, Pardi, Schaffner, \& Smyrk, 2007) 
La mayoría de los casos de PM no requieren tratamiento médico y solo se realiza un control evolutivo. Estos casos generalmente corresponden a la etapa temprana de la enfermedad, cuando predomina la necrosis grasa, cuando la sintomatología no presenta resolución espontánea, se ha sugerido el tratamiento con corticoides, ciclosporina, colchicina, tamoxifeno, progesterona, azatioprina, antibióticos y radioterapia con diferentes resultados, la cirugía solo se reserva para casos con retracción y oclusión intestinal, pudiéndose realizar derivaciones, resecciones o colostomías.

\section{CONCLUSIONES}

- Como conclusión, podemos decir que esta rara patología no tiene una etiología comprobada. Sus manifestaciones clínicas suelen ser inespecíficas, pero los hallazgos tomográficos, sin ser patognomónicos son de alta especificidad, haciendo cada vez más innecesaria la punción y el análisis histológico para la confirmación del diagnóstico. Su evolución es benigna, quedando la cirugía reservada solamente para las complicaciones.

- La SM es un trastorno idiopático poco común que afecta el mesenterio del intestino delgado con diversos grados de fibrosis, inflamación y necrosis grasa. $\mathrm{Su}$ presentación clínica es bastante variable y varía de asintomática a enfermedad debilitante. Aproximadamente el 50\% de los pacientes podría no necesitar ningún tratamiento. Sin embargo, en casos sintomáticos, el tratamiento debe adaptarse de acuerdo con la gravedad y el tipo de síntomas individuales.

- La SM es una entidad infrecuente que nos obliga a descartar una neoplasia asociada y que se trata en los casos sintomáticos. La cirugía está indicada para la obtención de biopsias, cuando no se pueden realizar de manera percutánea, y en los casos de oclusión intestinal.

\section{REFERENCIAS}

Aguilar Chalacán, M. F., Vela Benalcazar, C., Recalde, J. L., \& Negrete, R. (2021).

Mesenteritis esclerosante: diagnóstico y tratamiento. Scielo, 16. doi:10.30944/20117582.707

Akram, S., Pardi, D., Schaffner, J., \& Smyrk, T. (2007). Mesenteritis esclerosante: características clínicas, tratamiento y resultado en noventa y dos pacientes. $\begin{array}{lllll}\text { Gastroenterología } & \text { clínica } & y & \text { hepatología, } & 8 .\end{array}$ doi:https://doi.org/10.1016/j.cgh.2007.02.032 
Emory , T., Monihan, J. M., \& Carr , N. (2017). Mesenteritis esclerosante, paniculitis mesentérica y lipodistrofia mesentérica: ¿una sola entidad? PubMed, 13. doi:10.1097 / 00000478-199704000-00004

Labalde Martíneza, M., Guijarro, J., Pacheco Martínez, P., Fernández Escuder, B., Domingo Asenjo, T., Jiménez de los Galanes, S., . . Perea Fernández, F. (2016). Mesenteritis esclerosante como causa excepcional de dolor abdominal. Elsevier, 12. doi:10.1016/j.gastrohep.2010.12.004

Poniachik , J., \& Smok S, G. (2000). Mesenteritis retráctil Comunicación de cuatro casos. Scielo, 16. doi:http://dx.doi.org/10.4067/S0034-98872000001100010

Sharma, P., Yadav, S., Marie Needham, C., \& Feuerstadt, P. (02 de 2017). Mesenteritis esclerosante: una revisión sistemática de 192 casos. Springer, 10. doi:https://doi.org/10.1007/s12328-017-0716-5

Delgado Plasencia , 1., Rodríguez Ballester , López Tomassetti , F., A Hernández Morales, A., \& Carrillo Pallarés . (2007). Paniculitis mesentérica: experiencia en nuestro centro. PubMed, 14. doi:10.4321 / s1130-01082007000500010

Navarro, S., \& Rivas Rivas, M. (2018). Mesenteritis esclerosante "aparente enfermedad maligna": alternativa diagnóstica ante la presencia de dolor, ascitis y suboclusión intestinal. Sociedad Andaluza de Patologia DIgestiva, 11. doi:This article is available from https://www.sapd.es/revista/2018/41/2/07

Sánchez, A., Albertini, C., Cárdenas, K., Pérez, G., \& Bécker, J. (2002). Mesentiritis esclerosante. Presentación de un caso y revisión de la literatura. Bibliografia Latinoamericana, 15. 